\title{
Relationships between 9-Year-Olds' Math and Literacy Worries and Academic Abilities
}

\author{
Laura Punaro and Robert Reeve \\ Melbourne School of Psychological Sciences, University of Melbourne, VIC 3010, Australia \\ Correspondence should be addressed to Robert Reeve, r.reeve@unimelb.edu.au \\ Received 11 May 2012; Revised 16 August 2012; Accepted 30 August 2012 \\ Academic Editor: Helga Krinzinger
}

Copyright (C) 2012 L. Punaro and R. Reeve. This is an open access article distributed under the Creative Commons Attribution License, which permits unrestricted use, distribution, and reproduction in any medium, provided the original work is properly cited.

\begin{abstract}
We investigated whether 9-year-olds experience math and/or literacy worries and, if they do, whether it is related to problemsolving abilities. Fifty-eight children judged the correctness of math, literacy, and mental rotation problems that differed in difficulty and rated their worry level about the correctness of judgments. Nonverbal IQ, general math, and literacy abilities were also assessed. Results showed children's worry ratings varied as a function of task and problem difficulty. Latent class analyses of math and literacy worry ratings revealed high-, moderate- and low-worry subgroups in both domains. The high-worry math subgroup exhibited poorer math performance than the other math subgroups, demonstrating a link between math worry and math performance. No relationship was found between worry literacy subgroups and literacy performance. Moreover, no relationship was found between teachers' rating of children's academic and general worry and children's own worry ratings. The relevance of the findings for understanding math and literacy worry is discussed.
\end{abstract}

\section{Introduction}

Insofar as preadolescent children experience academicrelated anxieties/worries, it is unclear whether these effects are specific to an academic domain (e.g., math) exists in other domains (e.g., literacy), or, if they exist, affect abilities similarly across domains. Although it is assumed that the trepidation associated with math is greater than other academic domains $[1,2]$, research into anxiety problem-solving abilities associations in areas other than math is limited, even in older students [1]. Moreover, even though academic anxieties may exist in preadolescent children, it is unclear whether children would be able to accurately describe these experiences because of immature metacognitive abilities. The research reported herein investigates 9-year-olds' academic anxiety/worry-ability relationships in math, reading, and mental rotation (a nonacademic area), where children rate anxiety level immediately after solving a problem (We use the term "worry" rather than anxiety because pilot work suggested that children could readily provide examples of their worry experiences, but had more difficulty providing examples of anxiety experiences. Moreover, the term "worry" has occasionally been used as a way of describing "anxiety" to children (see $[2,42])$. Nevertheless, we also use the generic terms "math anxiety" and "literacy anxiety" because they are used in the literature). The aim is to better understand the nature of academic anxiety/worry-ability associations in preadolescent children.

Although most academic anxiety research focuses on math anxiety (MA), literacy anxiety may also exist. In one of the few studies on language anxiety, Carroll et al. [3] found that, compared to their literate peers, 5 - to 15 -year-olds with poor literacy tended to be more anxious about their language abilities. This is an important issue because MA might be an aspect of academic anxiety more generally. However, the reasons for literacy anxiety are not well understood, and the research considered herein mainly focuses on math anxiety/ worry performance relationships.

MA is thought to emerge in the preadolescent years, peak around Grades 9 to 10 , and not change thereafter $[4,5]$. It is associated with poor math achievement, as well as socioemotional and behavioral difficulties in high school students [1, 5-7]. Surprisingly little is known about the impact of math anxiety/worries on preadolescent children's math abilities [8, 
9]. Dowker [1] suggests that MA affects math performance only after Grade 4 (10 years old) - a claim supported by $\mathrm{Ma}$ [9] in his meta-analysis of research on the association between MA and math performance (note, however, Ma [9] only included three studies of children younger than 11 years old in his meta-analysis). Moreover, Krinzinger et al. [10] found no association between MA and arithmetic ability in 6- to 9-year-olds and suggested that the way young children are questioned about math-related anxieties may affect their responses. These findings suggest that young children are either not affected by math anxiety/worries, do not understand anxiety-related questions, or are unable to report their anxieties $[1,11,12]$.

It is often assumed that children are able to report anxiety states-a claim that overlooks limitations in children's metacognitive capabilities, which may affect their ability to answer questions about their own cognitive or socioemotional states [13]. In questionnaires typically used to assess MA, children are asked to reflect on general events (e.g., imagine being in a math lesson) and rate their anxiety, yet this may be beyond their cognitive capabilities $[9,14,15]$.

Some researchers have used simplified MA tasks to probe young children's anxiety states. Thomas and Dowker [16], for example, used a pictorial MA task in which 6- to 9-yearolds pointed to facial expressions to convey their emotional response to math events. The questions focused on children's perceived math ability, how much they enjoyed math tasks, and how worried they felt when they encountered difficult problems. Thomas and Dowker [16] found that their participants did not express anxiety or unhappiness at perceived math difficulties and concluded that MA was unrelated to poor math performance in young children. Gierl and Bisanz [12] also used a pictorial judgment paradigm to assess MA in 9- to 12 -year-olds. They found that 9-year-olds reported low levels of anxiety, while 12-year-olds reported higher anxiety levels. They concluded that MA increases as students mature and become more concerned about the consequences of success and/or failure. However, both groups of researchers asked probe questions about general retrospective events (e.g., experiences with mental calculation), rather than a specific problem solving event as they occurred. It is possible that the 9-year-olds in both studies lacked the abilities to reflect on general events [13].

The question of whether MA is a manifestation of general anxiety or is specific to math has been often debated. In a meta-analysis of MA research conducted on high school and college students, Hembree [5] reported moderate correlations ( 0.35 and 0.40$)$ between MA, general anxiety, trait anxiety, and state anxiety, and a stronger correlation (0.52) between MA and test anxiety. However, stronger correlations (0.5 to 0.8) have been found between MA measures (e.g., Math Anxiety Rating Scale and the Abbreviated Math Anxiety Scale), which suggests that MA is a specific anxiety-a claim supported by findings which show that general anxiety measures account for little of the variance in MA scores $[6,11]$. However, these analyses do not address whether MA is unique or a part of academic anxiety more generally. Findings from a study by Faust [17] suggest that MA is unique in older students, at least. He found that high MA students did not show anxiety on a verbal anagram task. A question of some interest is whether preadolescent children exhibit similar across-domain anxieties/worries. Indeed, Gierl and Bisanz [12] found that preadolescent children's MA ratings did not differ from their general school anxiety ratings, which could be interpreted as suggesting that children exhibit anxiety across academic domains.

A key aim of studying MA is to understand its impact on math abilities. Higher levels of MA are often linked to lower levels of math achievement $[2,7,18]$. However, the relationship between MA and math ability is complex. For instance, MA is often associated with avoidance behaviors, less time spent studying, and lower class engagement [10, 19], factors that can impact the acquisition of math skills. Poor performance by high MA students may also reflect the intrusion of worrisome thoughts while solving math problems. A reduced processing efficiencymodel was proposed by Eysenck and Calvo [20] who suggest that worries limit working memory capacity because they take up problemsolving resources $[4,10,11,21]$. Consistent with this claim, Ashcraft and Faust [22] found that MA had minimal impact on simple arithmetic problems, but had substantial impact on more difficult multiplication problems. However, not all researchers support the cognitive resources hypothesis. For arousal theorist, worry may motivate some students to increase efforts, while extreme worry may impede effort [2, 9]. Also, Wigfield and Meece [2] and Ho et al. [7] suggested that it is the affective-emotional component (i.e., tension, unpleasant physiological reactions) rather than the cognitive component that affects (correlates negatively) with math performance.

In the present study we investigate 9-year-olds' academic worry-performance associations, by probing their "worry" reactions using a "faces worry scale" immediately following problem solving (i.e., deciding whether two problems presented side by side represent the same solution to a problem). We are interested in whether worry judgments vary as a function of to-be-judged problem difficulty and problem correctness for math, literacy and, a nonacademic domain (mental rotation). If they do, it would confirm that children experience worry, whether or not they are able to report it. Of interest is whether math and language tasks elicit distinct patterns of worry judgments compared to the nonacademic task. If academic worries are unique, math and language worry judgments would be expected to differ from nonacademic worry judgments. Insofar as children's patterns of worry judgments differ, of interest is whether judgments are related to ability. On the basis of research with adolescents, it is expected that children's academic worry ratings would be associated with ability.

\section{Method}

2.1. Participants. Fifty-eight Grade 4 children (32 boys and 26 girls: $M=117.55$ months, $\mathrm{SD}=6.91$ ) from an urban primary school in a large Australian city participated. Common to Australian urban schools, the sample comprised children from diverse multicultural and socioeconomic backgrounds. All children had normal or correct-to-normal vision; and, 
according to school personnel, none had known learning difficulties. The research was, approved by, and conducted in accordance with, the authors' university human ethics committee requirements.

2.2. Materials and Procedures. To determine whether worry level differed as a function of math, literacy, or nonacademic (mental rotation) problems, children were presented two problems side by side from the three domains and (1) judged whether the solution to the pair of problems was the same or not and (2) rated their worry about the confidence they had in their decision using a face worry scale (described above) (see Table 1 for examples of pairs of to-be-judged problems for each of the three domains). Children judged 48 separate problems in each of the three domains $(n=144$ problems across the three domains) comprising pairs of to-be-judged problems that were easy or difficult to judge and for which the solutions were either equivalent or not $(n=12$ for each difficulty/equivalence combination within each domain: see Table 1 e.g., of these combination for each domain).

Children completed the Ravens Colored Progressive Matrices nonverbal IQ measures two weeks before judgment tasks [23]. They also completed a general numeracy and literacy worry test one week before completing judgment tasks. This task was used to (1) introduce children to the face worry judgment procedure, described above (see Figure 1), and (2) assess children's general retrospective worries. The term worry rather than anxiety was used in discussing faces with children because pilot work revealed it was more meaningful to them. Children were able to point to faces corresponding to "not worried at all," "a little bit worried" and "very worried" without hesitation and grasped the purpose of these procedures without difficulty.

Teachers rated children's general school-related anxiety prior to the judgment tasks. They also rated children's literacy and numeracy competency. Teachers were encouraged to consult children's formal test scores in making competency judgments-Australian children complete national numeracy and literacy tests throughout the primary (elementary) school years.

To avoid fatigue, the three domain judgment tasks were completed in three 15-minute sessions on consecutive days. Task procedures were explained before each session using four practice problems. Children were reintroduced to the faces worry scale (introduced one week earlier for use in the general numeracy and literacy worry judgment task) and encouraged to use the range of anxiety/worry faces to judge worry level if they felt it was appropriate to do so. Task order presentation was randomized across the three days; however within-task problems were presented in a fixed order.

The three domain judgment task problems were presented on a $15^{\prime \prime}$ laptop screen, and children judged whether to-be-judged problem pairs were equivalent by pressing the left-shift key (for equivalent/similar) or the right-shift key (for not equivalent/not similar). Task problems remained on the screen for a fixed time (math problems $=16$ seconds; language problems $=11$ seconds; mental rotation problems = 5 seconds). Presentation times were based on pilot data from a separate group of 10-year-olds and reflected the average

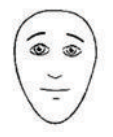

1

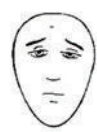

2

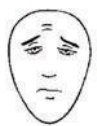

3

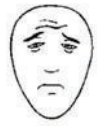

4

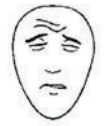

5

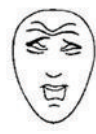

6
Figure 1: The faces worry scale.

time taken to complete easy and difficult problems. It was expected that children would have sufficient time to complete easy, but not hard problems, before problems disappeared from the screen. The perception of insufficient time to complete tasks is known to increase worry in children [24]. Although children could make a judgment before problems disappeared, few did so, and thus it was not possible to assess the function of response time.

As soon as children made a judgment, the faces worry scale appeared on the screen (see Figure 1 and description below). Children judged how worried they felt about the "correctness" of their equivalence judgment by pressing the numeric computer key corresponding to their worry face choice. Of interest were (1) judgment correctness and (2) worry ratings.

2.3. Judgment Tasks. For the math judgment task children judged whether two addition equations were equivalent. The problems were instances of associativity problems (e.g., $4+$ $3=3+2+2$ ) [25]. Difficulty was determined by the number of terms in the equation. Easy problems comprised equations of the form of " $\mathrm{a}+\mathrm{b}=\mathrm{b}+\mathrm{c}+\mathrm{d}$ " and difficult problems comprised equations of the form of $a+b+c=c+d+$ $\mathrm{e}+\mathrm{f}$. Research shows that 8 -year-olds are able to solve the easy associativity problems, and 10-year-olds are able to solve difficult problems [25]. Equations, terms comprised singledigit numbers between 2 and 9 (excluding doubles: e.g., $6+$ 6) and summed to 20 or less. Children's task was to judge whether both sides of the equal sign were equal or unequal. The task comprised 12 easy/same (correct), easy/different (incorrect), difficult/same, and difficult/different problems (see Table 1, e.g., of each).

The language judgment task comprised a synonym judgment test [26], designed to assess literacy (verbal reasoning, reading ability, vocabulary knowledge, and comprehension). Children were presented with two sentences, which differed in a single word and were asked to decide whether the two sentences were equivalent in meaning (e.g., "Elephants are larger than mice/Elephants are bigger than mice" and asked: "Do these sentences mean the same thing?"). Level of difficulty was determined by selecting synonym pairs that were appropriate for Grade 2 (easy) and Grade 4/5 (difficult). Example stimuli are presented in Table 1.

Quaiser-Pohl's [27] mental rotation test was used as the nonacademic task - we regarded it as a nonacademic measure because it requires skill not taught in school. It comprised a spatial ability measure designed for primary school aged children. Stimuli comprised colored pictures of two animals or humans, one of which was rotated around a central axis. Children judged whether the rotated figure was the same as 
TABLE 1: Example stimuli used in the math, literacy, and mental rotation tasks.

Task

\begin{tabular}{lll}
\multicolumn{1}{c}{ Math } & Literacy \\
\hline Easy/Same & $\begin{array}{l}\text { Peter RAISED/LIFTED the } \\
\text { trophy after winning the } \\
\text { Grand Final. } \\
\text { Mark is } \\
\text { STRONG/POWERFUL } \\
\text { enough to lift a truck. }\end{array}$ \\
\hline $3+7=2+3+5$ & $\begin{array}{l}\text { Mum's dinner was a } \\
\text { major FAILURE/SUCCESS. } \\
\text { Kate's pet turtle was very } \\
\text { LiGHT/HEAVY. }\end{array}$
\end{tabular}

$$
4+3+6=2+7+3+1
$$

Difficult/Same

$$
2+4+9=3+7+4+1
$$

CONDUCT/BEHAVIOUR

in the playground was

The policeman

PURSUED/CHASED the

thief through the alley.

atrocious.

\section{Steven waited}

$$
4+8+5+3=3+9+5
$$

PATIENTLY/RESTLESSLY

for his mother.

Roger was

$$
3+5+9=6+2+3+4
$$

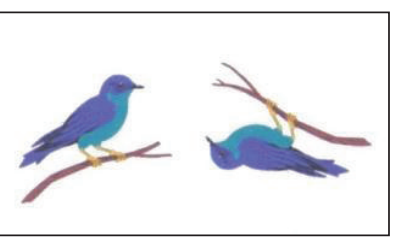

Difficult/Different
HOPEFUL/PESSIMISTIC

about getting the job. the target figure. Difficulty was determined by the rotation angle between the comparison pictures; easy problems were rotated less than 90 degrees (45 or 90 degrees), and difficult items were rotated more than 90 degrees (135 or 180 degrees).

Children's worry ratings for the math, literacy, and mental rotation tasks were computed for the problem difficulty and equivalence conditions (i.e., mean ratings were computed for the four within-task conditions for each of the tasks).

2.3.1. Worry Judgments. Worry judgment was assessed using the Faces Pain Anxiety Scale [28] (see Figure 2). The "faces scales" have been used in medical contexts to measure anxiety/worry [28-30]. The faces depict six different facial expressions in a graded sequence from nonanxious " 1 " to very anxious "6". The faces are claimed to represent approximately equal intervals in anxiety representations [28]. The Faces Anxiety Scale has a test-retest reliability of 0.79 [28] over a two-week period and correlates with similar scales $r=0.8$ on average [31]. The scale has been used with 3 -yearolds to older adults.

2.3.2. General Math and Literacy Worry. To assess children's general math and literacy worry, they were asked to rate their level of worry for math and literacy. Children rated "How worried do you usually feel when you are doing $x^{\prime \prime}$ ? ( $x=$ working on computers, sport, playing music, addition, subtraction, multiplication, reading, and writing stories). These questions were developed by the authors and served to (1) introduce children to the "faces worry scale" and (2) assess possible differences in children's general retrospective math and literacy worries. Children were introduced to the faces worry scales by judging their worry about working on computers, sports, and playing music. The purpose of using these neutral questions was to familiarize children with the worry judgment procedure and to answer questions if they arose. Following the so-called neutral questions, children made worry judgments about the addition, subtraction, multiplication, reading, writing stories questions. Children's math worry ratings were averaged across the three math items and literacy ratings were averaged across the two literacy ratings.

2.3.3. Teacher Judgment of Children's General Anxiety. Teachers completed the modified version of the School Anxiety Scale Teacher Report [32], which examines children's general and social anxiety in the school environments. This test has good test-retest reliability $(r=0.93)$ over an eight-week period), and is correlated $(r=0.83)$ with the Strengths and 


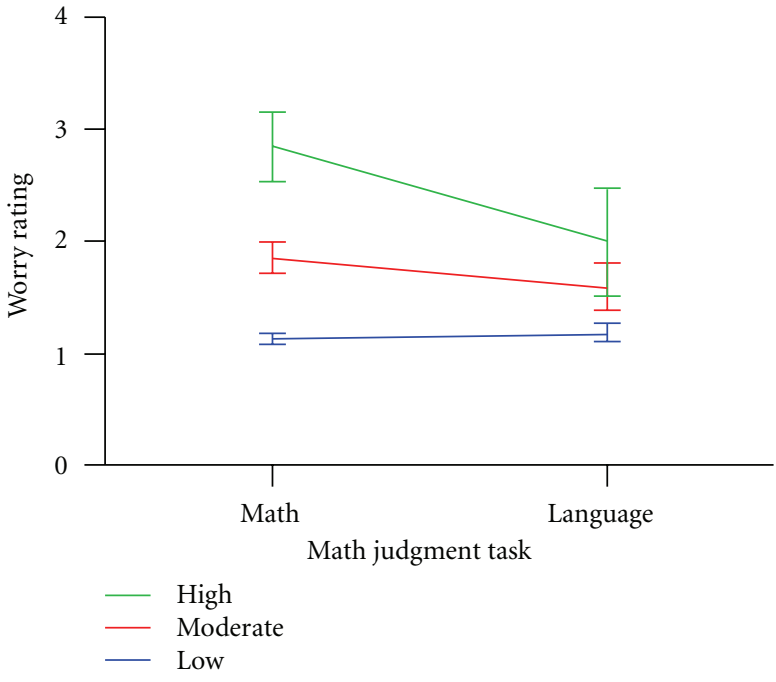

(a)

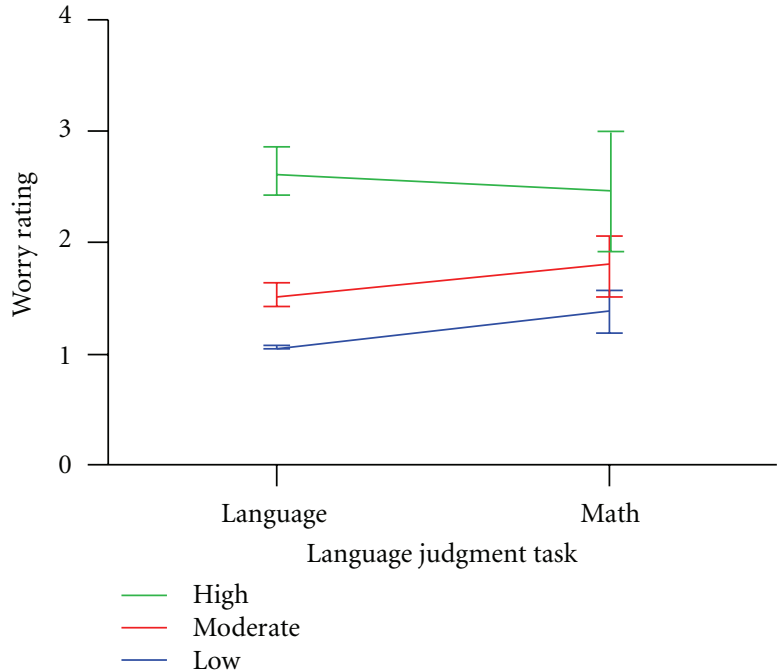

(b)

FIGURE 2: The relationships between math and literacy subgroup membership and literacy (a) and math (b) worry ratings, respectively.

Difficulties Questionnaire Internalizing Symptoms Scale, a widely used measure of teacher-rated anxiety. Seven general anxiety items were administered (e.g., "This child is afraid of making mistakes"; "This child worries about things"; "This child worries that (s)he will do badly at school"). Teachers rated anxiety on a four-point scale (0: never, 1 : sometimes, 2 : usually, 3: always). Teachers' ratings were averaged across the seven items.

2.3.4. Teacher Judgment of Children's Competency. Teachers rated children's ability in three areas - math, literacy (reading and writing), and general problem solving - on a five-point scale (1: poor, 2: not good, 3: average, 4: very good, and 5: superior). As noted earlier, teachers were encouraged to consult children's formal test results in rating their competencies.

2.4. Analytic Approach. Because we were not confident that the measurement scale properties of the math, literacy, and mental-rotation judgments tasks were sufficiently similar to combine them in analyses, we used separate ANOVAs to identify judgment correctness and worry rating for each task separately. Because we were also interested in identifying different patterns of worry as a function of within-task manipulations, we used latent class methods to isolate possible subgroup patterns of performances. Latent class (LC) methods [33] are a specific form of finite mixture modeling [34] approaches, which aim to uncover two or more subgroups of individuals characterized by distinct patterns of responding. A fundamental aspect of such models is the assumption of a categorical latent variable. The latent (unobserved) categories represent qualitatively different states of the underlying latent variable. Models are estimated with successively more latent classes, with the final model best capturing the structural representation of covariance between the indicator (observed) variables. Information criteria indices (e.g., AICc [35], CAIC [36]) were used to determine the best fitting model to the data, penalising increasingly complex specifications.

Unlike proximity-based cluster approaches (such as $K$-means), LC approaches offer a formal, model-based approach to the classification of individuals to unique clusters $[37,38]$. An important outcome of using this approach is probabilistic estimates of every individual's likelihood of belonging to each class of the latent variable, in this case, levels of worry.

Latent profile analysis (LPA) is a particular type of LC modelling [39]. What separates LPA from other LC models is that the profiles are formed based on continuous indicator variables; in this case, online worry judgments. Models were successively estimated from a two- to four-class specification. The relatively small sample size resulted in idiosyncratic classes beyond this range.

\section{Results}

Preliminary analyses showed no relationship between gender, Ravens measures, and any of the other measures of interest and so they were not included in analyses reported herein.

Three separate ANOVAs examined judgment accuracy of the to-be-judged problem pairs for the three tasks (math, literacy, and nonacademic tasks) as a function of the difficulty (easy or difficulty) and the similarity (same or different) of the two problems (see Table 2 for the means and standard deviations associated with these analyses). For math judgment accuracy, main effects of problem difficulty $\left(F(1,57)=80.83, P<0.001, \eta^{2}=0.59\right)$ and problem similarity $\left(F(1,57)=23.38, P<0.001, \eta^{2}=0.29\right)$ were found. In addition, the interaction between them was also significant $\left(F(1,57)=8.30, P<0.01, \eta^{2}=0.13\right)$. For literacy judgment accuracy, main effects of problem difficulty $\left(F(1,57)=349.00, P<0.001, \eta^{2}=0.86\right)$ and problem 
TABLE 2: Mean worry ratings and problem correctness (and standard deviations) for the math, literacy, and mental rotation tasks as a function of the difficulty and similarity of the to-be-judged problem pairs.

\begin{tabular}{lccc}
\hline \multirow{3}{*}{ Eame } & Different & Same & Different \\
\hline \multicolumn{4}{c}{ Math problems } \\
\hline $1.48(0.56)$ & $1.55(0.67)$ & $1.83(0.79)$ & $1.93(0.83)$ \\
$89.22(11.47)$ & $92.96(10.79)$ & $70.69(22.03)$ & $83.19(13.92)$ \\
\hline \multicolumn{4}{c}{ Literacy problems } \\
\hline $1.21(0.40)$ & $1.34(0.44)$ & $1.73(0.80)$ \\
$95.98(8.58)$ & $93.97(6.58)$ & $60.78(18.67)$ & $73.85(15.88)$ \\
\hline \multicolumn{4}{c}{ Mental rotation problems } \\
\hline $1.22(0.35)$ & $1.35(0.51)$ & $1.35(0.46)$ \\
$88.51(23.04)$ & $86.06(13.00)$ & $79.60(28.02)$ & $81.18(17.49)$ \\
\hline
\end{tabular}

similarity $\left(F(1,57)=7.93, P<0.001, \eta^{2}=0.12\right)$ were found. In addition, the interaction between them was also significant $\left(F(1,57)=23.21, P<0.01, \eta^{2}=0.29\right)$. For mental rotation nonacademic accuracy, only a main effect of difficulty was found $\left(F(1,57)=22.69, P<0.001, \eta^{2}=0.29\right)$.

Three separate ANOVAs also examined judgment worry ratings for the three tasks as a function of the problem difficulty (easy or difficulty) and the similarity (same or different) of the to-be-judged problem pairs. For math judgment worry ratings, main effects of difficulty $(F(1,57)=50.72, P<0.01$, $\left.\eta^{2}=0.47\right)$ and problem similarity $(F(1,57)=8.10, P<0.01$, $\left.\eta^{2}=0.12\right)$ were found. For literacy judgment worry ratings, only a main effect of difficulty was found $(F(1,57)=42.72$, $\left.P<0.01), \eta^{2}=0.43\right)$. In addition, the interaction between them was also significant $\left(F(1,57)=18.38, P<0.001, \eta^{2}=\right.$ $0.24)$. For mental rotation-nonacademic worry ratings, main effects of difficulty $\left(F(1,57)=7.92, P<0.01, \eta^{2}=0.12\right)$ and problem similarity $\left(F(1,57)=11.94, P<0.01, \eta^{2}=0.17\right)$ were found. In addition, the interaction between them was also significant $\left(F(1,57)=4.19, P<0.05, \eta^{2}=0.07\right)$.

These findings show that manipulating the difficulty and similarity of the to-be-judged problem pairs affected judgment accuracy and worry ratings for the math and literacy tasks; however, only task difficulty affected accuracy judgments in the nonacademic task.

The next set of analyses investigated the relationship between the math and literacy worry ratings and accuracy measures. Because of the relatively large math and literacy worry SD's, two separate latent profile analysis [39] were employed to identify possible worry subgroups in the two domains. Specifically, mean worry ratings for problem similarity and problem difficulty were used to identify cluster membership. Different criteria were used to identify the best fitting model (see Table 3). The AICc and CAIC values indicated a three-cluster solution for both the patterns of math and literacy worry judgments (see Table 3 ). In particular, the solutions revealed low-, moderate- and highworry subgroups for both domains. Classification errors, defining the degree of uncertainty between probabilistic cluster proportions and cluster membership using maximum
TABLE 3: Latent class analysis of the math and literacy worry ratings.

\begin{tabular}{lccccc}
\hline Cluster & LL & AICc & CAIC & BIC & CE (\%) \\
\hline \multicolumn{5}{c}{ Math worry clusters } \\
\hline 2-cluster & -104.83 & 224.95 & 295.68 & 278.68 & 0.62 \\
3-cluster & $-\mathbf{6 8 . 7 5}$ & $\mathbf{1 8 2 . 7 8}$ & $\mathbf{2 6 9 . 0 7}$ & $\mathbf{2 4 3 . 0 7}$ & $\mathbf{2 . 1 4}$ \\
4-cluster & -49.98 & 214.50 & 277.07 & 242.07 & 1.99 \\
\hline \multicolumn{7}{c}{ Literacy worry clusters } \\
\hline 2-cluster & -38.02 & 91.34 & 162.07 & 145.07 & 0.36 \\
3-cluster & $\mathbf{2 5 . 4 0}$ & $-\mathbf{5 . 5 1}$ & $\mathbf{8 0 . 7 8}$ & $\mathbf{5 4 . 7 8}$ & $\mathbf{0 . 7 6}$ \\
4-cluster & 45.81 & 22.92 & 85.50 & 50.50 & 1.87 \\
\hline
\end{tabular}

TABLE 4: Worry subgroups membership cross-classification across the math and literacy domains.

\begin{tabular}{lccc}
\hline \multirow{2}{*}{ Literacy worry subgroup } & \multicolumn{3}{c}{ Math worry subgroup } \\
& Low & Moderate & High \\
\hline Low & 18 & 8 & 1 \\
$\quad$ Count & 12.6 & 9.8 & 4.7 \\
$\quad$ Expected count & $2.9^{*}$ & -1 & $-2.5^{*}$ \\
$\quad$ Adjusted residual & & & \\
Moderate & 9 & 9 & 5 \\
$\quad$ Count & 10.7 & 8.3 & 4 \\
Expected count & -0.9 & 0.4 & 0.7 \\
Adjusted residual & & & \\
High & 0 & 4 & 4 \\
$\quad$ Count & 3.7 & 2.9 & 1.4 \\
Expected count & $-2.8^{*}$ & 0.9 & $2.6^{*}$ \\
Adjusted residual & & & \\
${ }^{*} P<0.05$. & & &
\end{tabular}

class assignment, were acceptably small, indicating that the subgroups were valid.

The relationship between low, moderate, and high subgroups across the math and language domains was significant $\left(\chi^{2}(4, N=58)=15.26, P<0.001\right)$, with a significant ordinal-by-ordinal association $(\gamma=0.67, P<0.001$ : see Table 4 for the cross-classification of the math and literacy subgroups). Table 4 reports differences between expected and observed cell frequencies, and the associated adjusted standardized residuals for significant differences among cells. The information in Table 4 could be interpreted as suggesting that there is a tendency to belong to similar worry groups in both the math and literacy domains; however, the information in Table 4 does not provide information about the within-domain differences on across-domain measures.

Figure 2 shows the mean worry ratings for math and literacy, respectively, as a function of the math and literacy subgroups. Figure 2 suggests a differential impact of subgroup membership on across-domain measures, which was confirmed by analyses of the across-domain worry ratings. Specifically, for the math worry subgroups, a significant interaction between math subgroup and literacy worry rating was found $\left(F(2,55)=12.47, P<0.001, \eta^{2}=0.31\right)$. However, for the literacy worry subgroups, no significant 
interaction between literacy subgroup and math worry ratings was found $\left(F(2,55)=2.26, P>0.05, \eta^{2}=0.08\right)$. (Note, only interactions are important here). These results suggest that literacy subgroup worry ratings might reflect general worry ratings, whereas the math worry subgroup ratings reflect unique within-domain differences in worry ratings.

The analyses reported thus far do not address directly the question of whether worry subgroup memberships predict judgment accuracy. These relationships are reported in Table 5, which appears to show that judgment accuracy is indeed associated with worry. In the next set of analyses we report findings from regression analyses in which withindomain worry subgroup membership respectively predicted within-domain judgment accuracy over and above the effect of teachers competence ratings and children's own general worry ratings. The latter two factors are included in the analyses to determine whether teachers' assessment of children's competence and children's general retrospective worry judgments moderate the relationship between worry subgroup membership and judgment accuracy.

Two separate multiple regression analyses were conducted predicting math and literacy correctness judgments, respectively, subgroup membership was entered as a dummy variable (the low-worry subgroup was used as the reference category in both analyses). (Note: we only used the accuracy for difficult judgments because of ceiling effects associated with easy accuracy judgments-see Table 5).

VIF scores were low, suggesting that multicollinearity was not an issue in the analyses. The overall model for math problem judgments was significant $(F(4,53)=5.50, P<$ $0.01, R^{2}=0.29$ ) (see Table 6 for the regression parameters). Two significant predictors emerged: teacher rating of math competency and worry subgroup membership. Specifically, teacher math competency ratings predicted math task judgment performance $(\beta=0.46, P<0.01)$ and membership of the high worry subgroup predicted poorer math task judgment performance, relative to belonging to the lowworry subgroup $(\beta=-0.33, P<0.05)$. Retrospective worry ratings did not predict difficult math judgment performance. The overall model for literacy problem judgments was significant $\left(F(4,53)=2.97, P<0.05, R^{2}=0.18\right.$ ) (see Table 7 for the regression parameters). Only children's own retrospective literacy worry rating predicted literacy problem judgment correctness $(\beta=0.46, P<0.01)$. It is evident that different factors predict math and literacy correctness judgments.

\section{Discussion}

The aim of the research was twofold. The first was to determine whether 9-year-olds report differences in math, literacy, and nonacademic worry immediately after solving a problem using the "faces worry scale." The second was to see whether differences in worry ratings were related to problem solving. Three findings are of note. First, worry ratings were higher for more difficult math, language, and mental rotation problems, indicating that children were sensitive to task demands. Second, overall, worry levels were higher for the math problems, compared to language and nonacademic (mental rotation) problems. Three meaningful worry subgroups were identified from worry ratings for both the math and the language tasks (but not for the nonacademic mental rotation task). Importantly, children with high math worry were less successful in making correct math judgments compared to children with low math worry. As noted earlier, neither gender nor general ability effects were observed.

The faces worry scale was used (1) immediately after children made judgment about the equivalence of pairs of math, literacy, and mental rotation problems and (2) to assess children's general math and literacy worries. Children's worry ratings increased as math and literacy judgment task difficulty increased, which shows that children were sensitive to task difficulty (however, worry ratings did not increase as the difficulty of the mental rotation problems increased). This particular finding stands in contrast to Thomas and Dowker's [16] and Gierl and Bisanz's [12] findings that most 6- and 9-year-olds' did not exhibit math performancerelated anxiety. Our finding adds weight to claims that math anxiety symptoms may be displayed in primary-aged children $[9,10,40]$ and is inconsistent with claims that math anxiety symptoms only appear in late primary/elementary school students $[1,11]$. It is possible of course that the latter studies may have underestimated young children's academic anxieties because of difficulties associated with assessing affective states retrospectively [13]. In the present study, this difficulty was minimized by asking children about their worry immediately after solving a problem. Nevertheless, it is also evident that children in the current study were able to use the "faces worry scale" to rate general math and literacy worries.

MA is considered a unique anxiety linked specifically to math [5]. Math is thought to elicit greater worry than other academic domains [1, 17]. Wigfield and Meece [2] suggest that math is particularly anxiety provoking because of its emphasis on precision, logic, and problem solving. While our findings support this claim (worry ratings were higher for math than language), it is evident that 9-year-olds also experienced language worries. This finding suggests that both math and language elicit worry reactions. Interestingly, a similar differential in worry ratings was found in the general academic worry ratings: math problems were rated as more worrisome than literacy problems.

It is noteworthy that associations were found between the math and language subgroups and other tasks. It is evident that (1) children in the low math anxiety/worry subgroup showed equally low levels of worry on all three judgment tasks; (2) children in the high math anxiety/worry subgroup found the math task more worrisome than the language task; (3) some children only showed high levels of worry on the math task, but not on other tasks. The latter finding is important because it provides support for the specificity of math anxiety.

The fact that some children reported high levels of worry in the literacy judgment task suggests literacy per se may evoke anxiety. Indeed, the link between reading difficulties and anxiety symptoms has been noted by researchers [41]. It is assumed that anxiety arises in response to experiences 
TABLE 5: Worry ratings and problems corrects as a function of math and literacy worry groups.

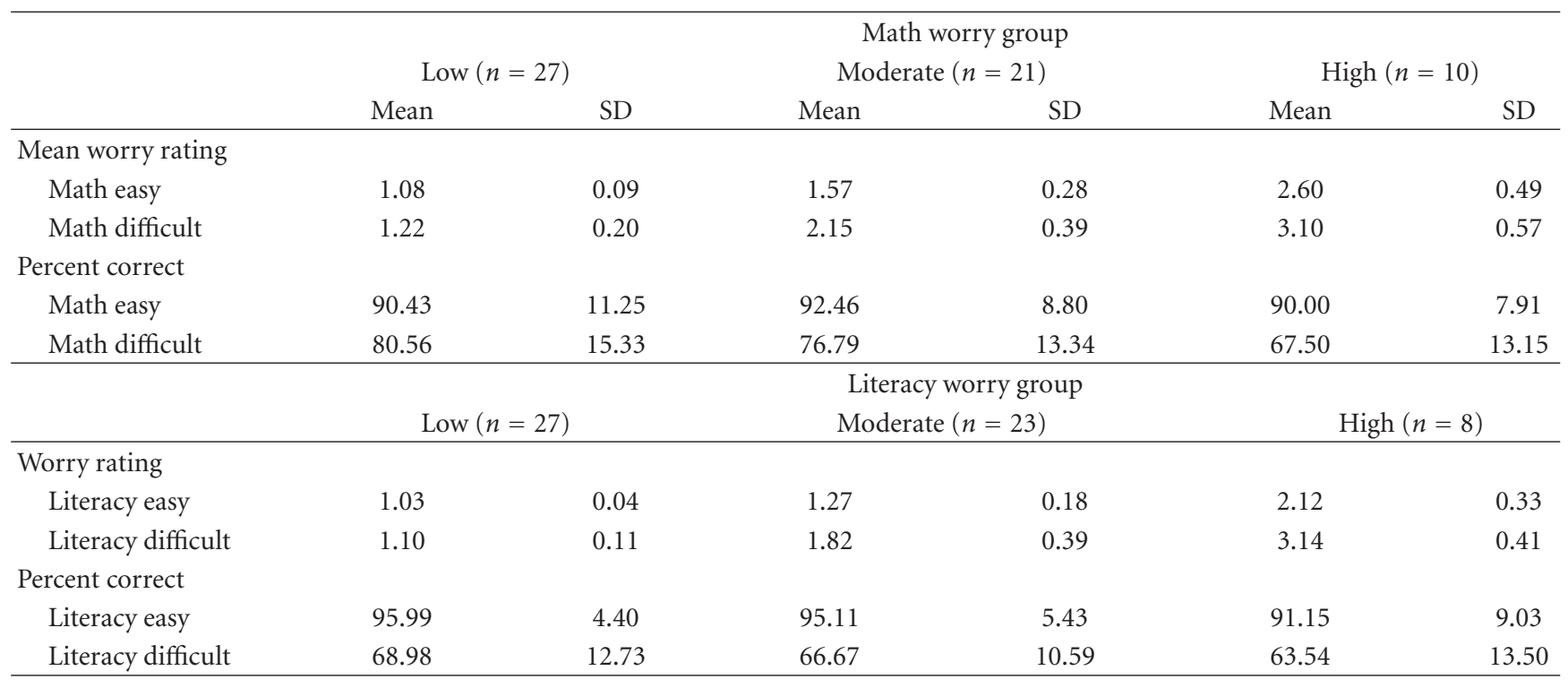

TABLE 6: Predicting math judgment correctness: multiple regression parameters.

\begin{tabular}{|c|c|c|c|c|c|}
\hline & $B$ & Std. Error & $\beta$ & $t$ & Sig. \\
\hline (Constant) & 57.09 & 8.89 & & 6.42 & 0.000 \\
\hline $\begin{array}{l}\text { Moderate worry } \\
\text { subgroup }^{1}\end{array}$ & -0.09 & 4.20 & -0.00 & -0.02 & 0.983 \\
\hline High worry subgroup ${ }^{1}$ & -12.71 & 5.13 & -0.33 & -2.48 & 0.016 \\
\hline $\begin{array}{l}\text { Teacher rating math } \\
\text { competency }\end{array}$ & 6.73 & 1.86 & 0.46 & 3.61 & 0.001 \\
\hline $\begin{array}{l}\text { Children's retrospective } \\
\text { math worry }\end{array}$ & 0.11 & 2.79 & 0.01 & 0.04 & 0.968 \\
\hline
\end{tabular}

${ }^{1}$ Reference category is low subgroup.

TABLe 7: Predicting literacy judgment correctness: multiple regression parameters.

\begin{tabular}{|c|c|c|c|c|c|}
\hline & $B$ & Std. Error & $\beta$ & $t$ & Sig. \\
\hline (Constant) & 45.65 & 8.91 & & 5.13 & 0.000 \\
\hline $\begin{array}{l}\text { Moderate worry } \\
\text { subgroup }^{1}\end{array}$ & -2.14 & 3.18 & -0.09 & -0.67 & 0.505 \\
\hline High worry subgroup ${ }^{1}$ & -2.86 & 4.62 & -0.08 & -0.62 & 0.539 \\
\hline $\begin{array}{l}\text { Teacher rating literacy } \\
\text { competency }\end{array}$ & 1.61 & 2.73 & 0.08 & 0.59 & 0.559 \\
\hline $\begin{array}{l}\text { Child retrospective } \\
\text { reading worry }\end{array}$ & 6.01 & 1.89 & 0.42 & 3.19 & 0.002 \\
\hline
\end{tabular}

${ }^{1}$ Reference category is low subgroup.

of failure rather than literacy anxiety per se. Our results suggest that literacy anxiety is not necessarily a reflection of poor ability, but can arise in children with age-appropriate reading skills. Indeed, our results showed that children in the high language worry subgroup also found the math task worrisome (see Figure 2). However, it should be noted that children in the high math worry subgroup did find the language task worrisome. The difference in the direction of these relationships suggests that language worry might reflect general academic-related worries, whereas math worry is unique to math.

Interestingly, teachers' ratings of children's general anxiety (as assessed on the School Anxiety Scale) were unrelated to children's immediate or general worry ratings. This finding is consistent with previous research with adolescent and adult samples $[4,5,11]$.

Some researchers have identified math anxiety in young children that was unrelated to math performance $[10,40]$. The failure to find a relationship has led some researchers to question the existence of a math anxiety performance link in children [1,9]. However, Krinzinger et al. [10] suggest that the failure to find a math anxiety-performance link might be due to methodological issues rather than an absence of math anxiety. In the present study, we found meaningful relationships between worry ratings and problem solving judgments. Similar to Krinzinger et al. [10, 42] we assessed "worry" using a faces rating scale. However, there are two key differences between our scale and the scale used by Krinzinger et al. [10, 42]. First, children rated worry immediately after making problem solving judgments. Second, our worry scale was unipolar in nature (neutral face to worried face); in contrast, Krinzinger et al's [10, 42] scale was bipolar in nature (from happy to neutral to worried). Tomlinson et al. [43] suggest that showing a range of emotions on the same scale that require anxiety judgment maybe difficult for children to understand. Nevertheless, it is evident that the children in our study were also able to use our faces worry scale to rate their worry about general math and literacy abilities.

It has been claimed that worry interferes with cognition because it focuses attention on intrusive thoughts rather than the task at hand $[10,11,21]$. Further, MA is claimed to 
reduce working memory capacity, which in turn affects math performance. Two findings from the present study are relevant here. First, a relationship between worry subgroup and performance emerged in comparing performance among the three math worry subgroups. Children in the high-worry group made fewer correct judgments than children in the low-worry group judging difficult problems. In the literacy task, overall worry groups differed in judgment accuracy, although no specific subgroup differences were identified. However, recent research suggests that the relationship between numerical cognition and MA is complex. For example, individuals with high MA also possess poor (1) spatial processing ability [42, 44], (2) dot enumeration [45], and (3) symbolic number comparison abilities [46]. These findings suggest that much more work needs to be undertaken to unpack the relationships between MA, numerical cognitive abilities, and math performance.

The present research findings have implications for educational practitioners. In particular, our findings show that it is possible to identify "at risk worry"-children earlier than previously thought. Moreover, it might be valuable to inform teachers of the consequences of academic-related worry on performance so that they can help at risk children minimize worry. Future research should focus on the impact on children's academic performance of reducing worry (see [47], e.g., on reducing, alleviating, and managing anxiety in students).

Although the current research findings provide evidence for a relationship between academic-related worry and the associated academic competencies, it is limited in at least three respects. First, the sample size $(n=58)$ was relatively small and limited possible analyses. In particular, with a larger sample we would have been able to investigate the direction of causal effects more explicitly. Second, it is evident the future research should examine the relationships between general cognitive factors (e.g., different measures of working memory), specific aspects of numerical cognition (e.g., dot enumeration, number comparison), problem solving, and worry. Third, in the present study, we were unable to examine the sequelae associated with response times. Studies have shown that time pressure affects anxiety ratings, and manipulating time requirements might shed more information about the relationships between worry, problem solving, and cognition.

Interestingly, similar to other researchers who have examined MA in preadolescent children, we found no sex differences in worry judgments $[2,12,40]$. The absence of a gender effect stands in contrast to research with adolescent samples $[9,48]$ in which gender differences have been found in MA. The developmental context in which MA gender effects emerge requires research.

The present study investigated 9-year-olds' worries using a sensitive measure in two academic domains-math and language- and the relationship between worry and problem solving ability. Results showed that children's worry varied with problem difficulty, and that math and literacy worry differed from nonacademic worry. Moreover, math worry predicted math ability, and a negative association was observed between high math worry and math performance. It is evident that the faces worry scale is a sensitive worry measure for assessing both immediate problem solving and more general academic worries. Nevertheless, more research is needed to investigate worry ratings across a broader range of academic-related problems that differ in difficulty, as well as to investigate how worry ratings change over time.

\section{Acknowledgments}

The authors would like to thank our colleagues Jacob Paul and Kelly Trezise for their willingness to discuss the research described herein: the paper benefitted greatly from their input. They would also like to thank Dr. Krinzinger and three anonymous reviewers for the helpful comments made on an earlier draft of the paper.

\section{References}

[1] A. Dowker, Individual Differences in Arithmetic: Implications For Psychology, Neuroscience and Education, Psychology Press, Hove, UK, 2005.

[2] A. Wigfield and J. L. Meece, "Math anxiety in elementary and secondary school students," Journal of Educational Psychology, vol. 80, no. 2, pp. 210-216, 1988.

[3] J. M. Carroll, B. Maughan, R. Goodman, and H. Meltzer, "Literacy difficulties and psychiatric disorders: evidence for comorbidity," Journal of Child Psychology and Psychiatry and Allied Disciplines, vol. 46, no. 5, pp. 524-532, 2005.

[4] M. H. Ashcraft and A. M. Moore, "Mathematics anxiety and the affective drop in performance," Journal of Psychoeducational Assessment, vol. 27, no. 3, pp. 197-205, 2009.

[5] R. Hembree, "The Nature, effects, and relief of Mathematics Anxiety," Journal For Research in Mathematics Education, vol. 21, pp. 33-46, 1990.

[6] M. H. Ashcraft and K. S. Ridley, "Math anxiety and its cognitive consequences," in Handbook of Mathematical Cognition, J. I. D. Campbell, Ed., pp. 315-327, Psychology Press, New York, NY, USA, 2005.

[7] H. Z. Ho, D. Senturk, A. G. Lam et al., "The affective and cognitive dimensions of math anxiety: a cross-national study," Journal for Research in Mathematics Education, vol. 31, no. 3, pp. 362-379, 2000.

[8] W. S. Bush, "Factors related to changes in elementary students' mathematics anxiety," Focus on Learning Problems in Mathematics, vol. 13, pp. 33-43, 1991.

[9] X. Ma, "A meta-analysis of the relationship between anxiety toward mathematics and achievement in mathematics," Journal for Research in Mathematics Education, vol. 30, no. 5, pp. 520-540, 1999.

[10] H. Krinzinger, L. Kaufmann, and K. Willmes, "Math anxiety and math ability in early primary school years," Journal of Psychoeducational Assessment, vol. 27, no. 3, pp. 206-225, 2009.

[11] M. H. Ashcraft, J. A. Krause, and D. R. Hopko, "Is math anxiety a mathematical learning disability?" in Why is Math So Hard For Some Children? the Nature and Origins of Mathematical Learning Difficulties and disabilities, D. B. Berch and M. M. Mazzocco, Eds., pp. 329-348, Paul H. Brookes Publishing, Baltimore, Md, USA, 2007.

[12] M. J. Gierl and J. Bisanz, "Anxieties and attitudes related to mathematics in Grades 3 and 6," Journal of Experimental Education, vol. 63, pp. 139-158, 1995. 
[13] M. A. Campbell and R. M. Rapee, "Current issues in the assessment of anxiety in children and adolescents: a developmental perspective," Behaviour Change, vol. 13, no. 3, pp. 185-193, 1996.

[14] C. R. Brewin, B. Andrews, and I. H. Gotlib, "Psychopathology and early experience: a reappraisal of retrospective reports," Psychological Bulletin, vol. 113, no. 1, pp. 82-98, 1993.

[15] P. Harris, Children and Emotion: The Development of Psychological Understanding, Basil Blackwell, Oxford, UK, 1989.

[16] G. Thomas and A. Dowker, "Mathematics anxiety and related factors in young children," in Proceedings of the British Psychological Society Developmental Section Conference, Bristol, UK, September 2000.

[17] M. W. Faust, Analysis of physiological reactivity in mathematics anxiety [doctoral dissertation], Bowling Green State University, Columbus, Ohio, USA, 1992.

[18] E. Satake and P. P. Amato, "Mathematics anxiety and achievement among Japanese elementary school students," Educational and Psychological Measurement, vol. 55, pp. 1000-1008, 1995.

[19] F. Yüksel-Şahin, "Mathematics anxiety among 4thand 5th Grade Turkish elementary school students," International Electronic Journal of Mathematics Education, vol. 3, pp. 179-192, 2008.

[20] M. W. Eysenck and M. G. Calvo, "Anxiety and performance: the processing efficiency theory," Cognition and Emotion, vol. 6, pp. 409-434, 1992.

[21] X. Ma and J. Xu, "The causal ordering of mathematics anxiety and mathematics achievement: a longitudinal panel analysis," Journal of Adolescence, vol. 27, no. 2, pp. 165-179, 2004.

[22] M. H. Ashcraft and M. W. Faust, "Mathematics anxiety and mental arithmetic performance: an exploratory investigation," Cognition and Emotion, vol. 8, no. 2, pp. 97-125, 1994.

[23] J. C. Raven, J. H. Court, and J. Raven, Manual For Raven's Progressive Matrices and Vocabulary scales, Lewis, London, UK, 1986.

[24] R. F. Salame, "Test anxiety: its determinants, manifestations, and consequences," in Advances in Test Anxiety Research, $\mathrm{H}$. M. van der Ploeg, R. Schwarzer, and C. D. Spielberger, Eds., pp. 83-119, Lawrence Erlbaum Associates, Hillside, NJ, USA, 1984.

[25] K. H. Canobi, R. A. Reeve, and P. E. Pattison, "Young children's understanding of addition concepts," Educational Psychology, vol. 22, no. 5, pp. 513-532, 2002.

[26] W. A. Garnham, J. Brooks, A. Garnham, and A. M. Ostenfeld, "From synonyms to homonyms: exploring the role of metarepresentation in language understanding," Developmental Science, vol. 3, no. 4, pp. 428-441, 2000.

[27] C. Quaiser-Pohl, "The mental cutting test "schnitte" and the mental rotation rotation test-two new measures to assess spatial ability," International Journal of Testing, vol. 3, pp. 219231, 2003.

[28] D. Bieri, R. A. Reeve, G. D. Champion, L. Addicoat, and J. B. Ziegler, "The faces pain scale for the self-assessment of the severity of pain experienced by children: development, initial validation, and preliminary investigation for ratio scale properties," Pain, vol. 41, no. 2, pp. 139-150, 1990.

[29] H. R. Chapman and N. Kirby-Turner, "Visual/verbal analogue scales: examples of brief assessment methods to aid management of child and adult patients in clinical practice," British Dental Journal, vol. 193, no. 8, pp. 447-450, 2002.

[30] K. E. Howard and R. Freeman, "Reliability and validity of a faces version of the modified child dental anxiety scale,"
International Journal of Paediatric Dentistry, vol. 17, no. 4, pp. 281-288, 2007.

[31] J. N. Stinson, T. Kavanagh, J. Yamada, N. Gill, and B. Stevens, "Systematic review of the psychometric properties, interpretability and feasibility of self-report pain intensity measures for use in clinical trials in children and adolescents," Pain, vol. 125, no. 1-2, pp. 143-157, 2006.

[32] H. J. Lyneham, A. K. Street, M. J. Abbott, and R. M. Rapee, "Psychometric properties of the school anxiety scale-teacher report (SAS-TR)," Journal of Anxiety Disorders, vol. 22, no. 2, pp. 292-300, 2008.

[33] L. A. Goodman, "Latent class analysis: the empirical study of latent types, latent variables, and latent structures," in Applied Latent Class Analysis, J. A. Hagenaars and A. L. McCutcheon, Eds., pp. 3-53, Cambridge University Press, Cambridge, UK, 2002.

[34] G. J. McLachlan and D. Peel, Finite Mixture Models, John Wiley \& Sons, New York, NY, USA, 2000.

[35] C. M. Hurvich and C. L. Tsai, "Regression and time series model selection in small samples," Biometrika, vol. 76, no. 2, pp. 297-307, 1989.

[36] H. Bozdogan, "Model selection and Akaike's Information Criterion (AIC): the general theory and its analytical extensions," Psychometrika, vol. 52, no. 3, pp. 345-370, 1987.

[37] J. Magidson and J. K. Vermunt, "Latent class clustering as a probabilistic extension of K-Means," Quirk's Marketing Research Review, vol. 20, pp. 77-80, 2002.

[38] J. Magidson and J. K. Vermunt, "Latent class models for clustering: a comparison with K-means," Canadian Journal of Marketing Research, vol. 20, pp. 36-43, 2002.

[39] L. M. Collins and S. T. Lanza, Latent Class and Latent Transition Analysis For the Social, Behavioral, and Health Sciences, John Wiley \& Sons, New York, NY, USA, 2010.

[40] C. B. Young, S. S. Wu, and V. Menon, "The neurodevelopmental basis of math anxiety," Psychological Science, vol. 23, pp. 492-501, 2012.

[41] J. M. Carroll and J. E. Iles, "An assessment of anxiety levels in dyslexic students in higher education," British Journal of Educational Psychology, vol. 76, no. 3, pp. 651-662, 2006.

[42] H. Krinzinger, G. Wood, and K. Willmes, "What accounts for individual and gender differences in the multi-digit number processing of primary school children?" Zeitschrift für Psychologie, vol. 220, pp. 78-79, 2012.

[43] D. Tomlinson, C. L. von Baeyer, J. N. Stinson, and L. Sung, "A systematic review of faces scales for the self-report of pain intensity in children," Pediatrics, vol. 126, no. 5, pp. e1168e1198, 2010.

[44] E. A. Maloney, S. Waechter, E. F. Risko, and J. A. Fugelsang, "Reducing the sex difference in math anxiety: the role of spatial processing ability," Learning and Individual Differences, vol. 22, no. 3, pp. 380-384, 2012.

[45] E. A. Maloney, E. F. Risko, D. Ansari, and J. Fugelsang, "Mathematics anxiety affects counting but not subitizing during visual enumeration," Cognition, vol. 114, no. 2, pp. 293-297, 2010.

[46] E. A. Maloney, D. Ansari, and J. A. Fugelsang, "The effect of mathematics anxiety on the processing of numerical magnitude," Quarterly Journal of Experimental Psychology, vol. 64, no. 1, pp. 10-16, 2011.

[47] E. A. Maloney and S. L. Beilock, "Math anxiety: Who has it, why it develops, and how to guard against it," Trends in Cognitive Sciences, vol. 16, no. 8, pp. 404-406, 2012. 
[48] X. Ma and F. Cartwright, "A longitudinal analysis of gender differences in affective outcomes in mathematics during middle and high school," School Effectiveness and School Improvement, vol. 14, no. 4, pp. 413-439, 2003. 


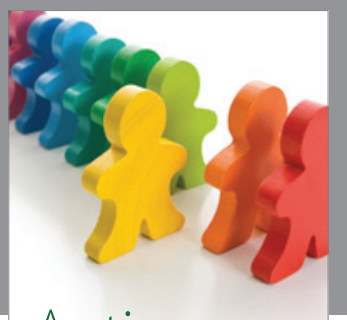

Autism

Research and Treatment
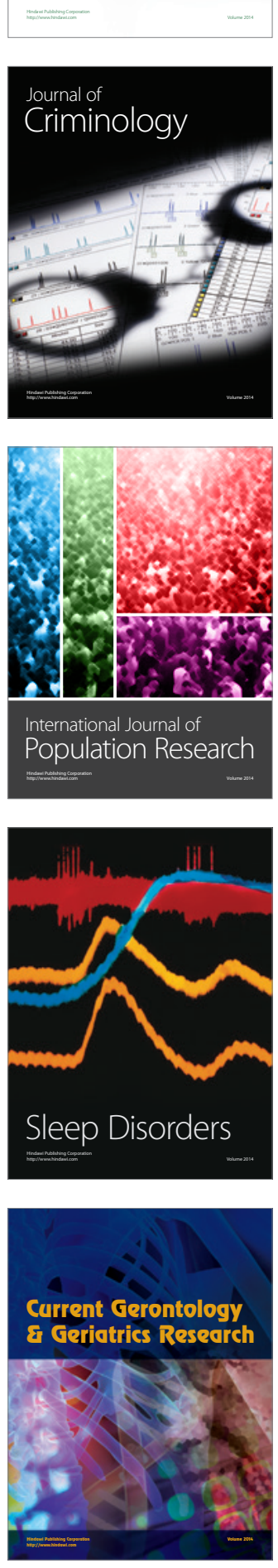
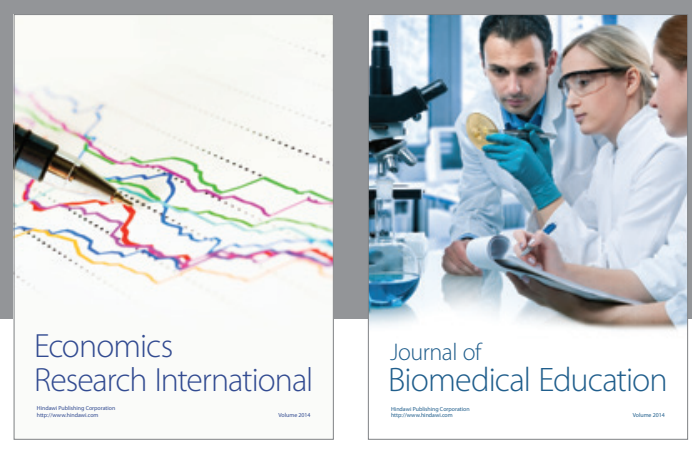

Journal of

Biomedical Education

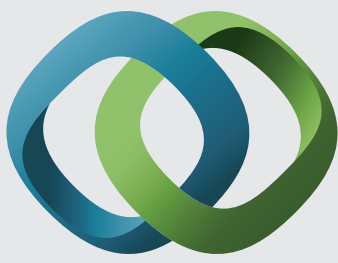

\section{Hindawi}

Submit your manuscripts at

http://www.hindawi.com
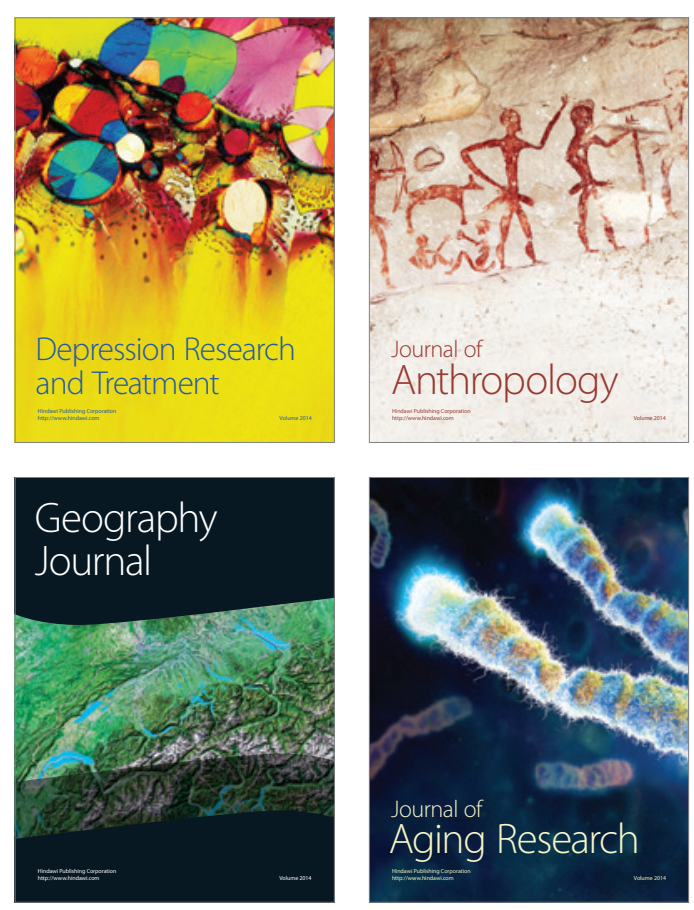

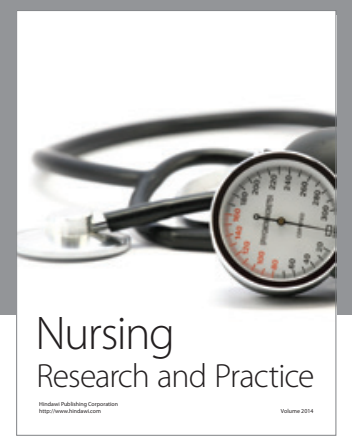

Nursing

Research and Practice

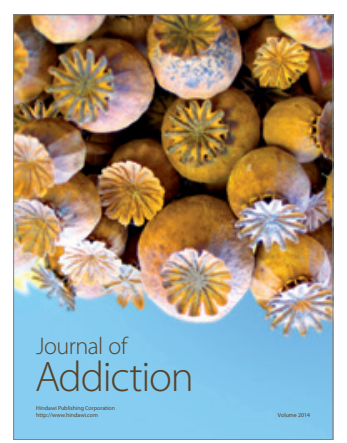

Child Development

Research

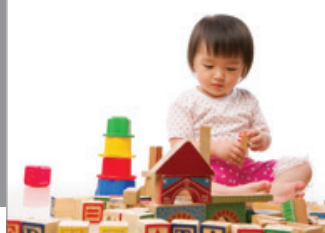

迥
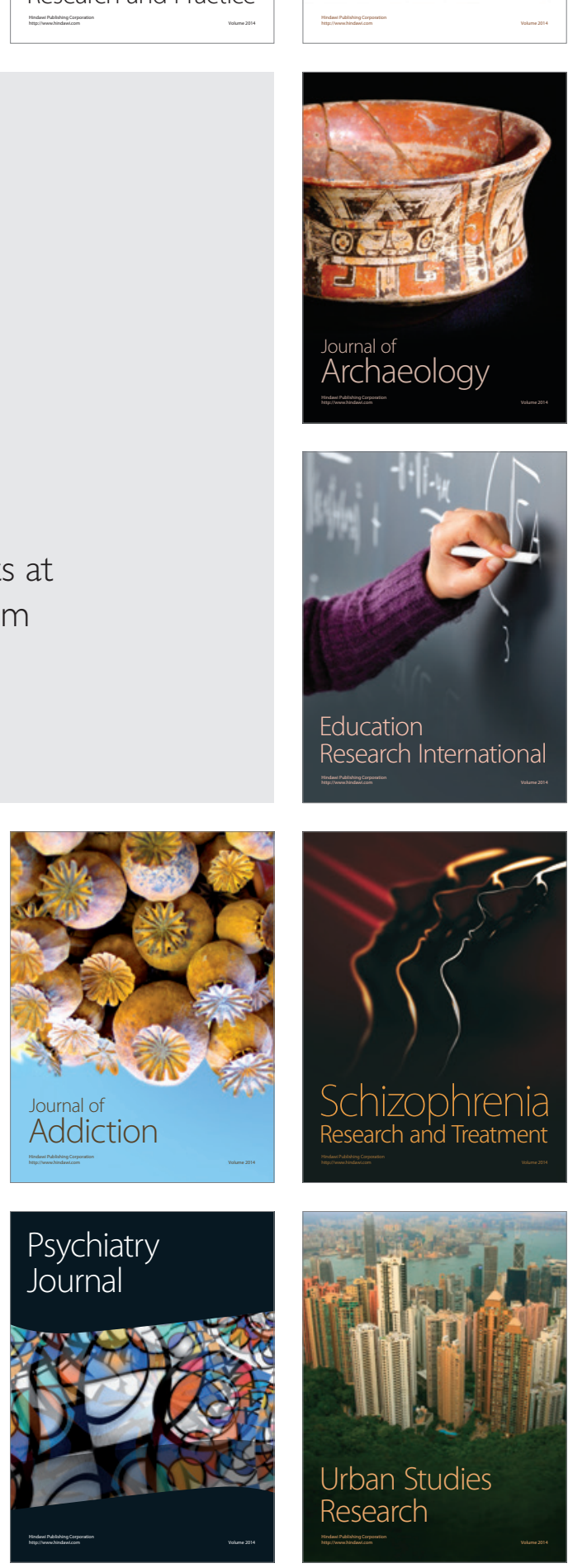